\title{
The Function of Human Movement Science to Competitive Sports
}

\author{
Zimin Chang, Wenwen Wang
}

Nanchang Institute of Science \&Technology,Nanchang 330108,China

Keywords: Human movement science; competitive sports; scientific selection

\begin{abstract}
Through the method of literature, a relevant part of the contents of human movement science and sports are analyzed. This part includes athlete scientific selection, training monitoring, physical recovery and nutritional supplements, sports injuries and medical supervision, monitoring and elimination of fatigue and anti doping research, through the analysis of these aspects in sports training and the relationship with human movement science, we found that human movement science plays an important role in competitive sports, it plays an important role in improving the performance of sports, and it is an important science to support the development of competitive sports.

Human movement science is the study of the relationship between sports and human body. Including sports anatomy, sports physiology, sports biochemistry, health rehabilitation and sports medicine and other disciplines. It is through the experts brewing, after discussion in 1997 on the basis of the original subject catalog to broaden the scope of the professional. Competitive sports is also known as athletic sports, it is an important part of sports, which is the main feature of sports competition, to create excellent performance, to win the game as the main goal of social sports activities. An important research field of competitive sports including the scientific selection of athletes, the theories and methods of training, scientific training and monitoring function evaluation, physical recovery and nutritional supplements, exercise psychological theories and methods, sports injuries and medical supervision, prevention and monitoring of fatigue, anti-doping research, information technology services and competitive sports management science etc.. The scientific selection of athletes, training monitoring and functional assessment, physical recovery and nutritional supplements, sports injuries and medical supervision, prevention and monitoring of fatigue, anti-doping of six blocks is closely linked with human movement science.
\end{abstract}

\section{Human movement science and scientific selection of athletes}

The scientific selection is according to the characteristics and requirements of different sports, by means of modern science, through the objective index test, comprehensive evaluation and prediction, the congenital advantages, suitable for a movement of people from selection, systematic training, a process of continuously monitoring and its development trend.

Sports is a frontier science, which is based on multi-disciplinary. It is precisely because of the progress of modern science and technology, sports science research can be developed in depth, it provides a theoretical basis for the scientific selection of athletes. It is the theory and method system of human genetics, sports training, sports anthropology, sports physiology, sports measurement and so on. These are some of the branches of sports science. For sports training, it can provide effective data. It provides an important basis for scientific and reasonable strength training, provides objective conditions for improving sports performance, and provides reference for scientific selection of sports talents. But our current material and scientific research strength is weak, the main athletes on the athletes' body shape test, exercise biochemistry, sports psychology, the parties 
and the obligation of supervision and monitoring, provide a great reference value for sports training, which has achieved a certain effect. But in the future it should be more open to the field of scientific research, and constantly strengthen the study of human movement science, one of the data widely used in practice, service for front-line coaches and athletes. Therefore, human movement science and scientific selection of athletes have directly relation.

\section{Human movement science and training monitoring, function evaluation}

The application of biochemical indicators to monitor the training of athletes, to ensure the scientific training, which is a guarantee of performance improvement. The commonly used monitoring indicators include hemoglobin, urea nitrogen, creatine kinase, immunoglobulin and other biochemical indicators. According to these indexes, the training process is monitored and evaluated, the difference is bigger, establishes the athlete training monitoring individual file, carries on the longitudinal comparison and the analysis; for the individual target weak athlete, must pay attention to the active prevention and the treatment. The content of this part is the research of human movement science category, with biological and medical technology, the research in this aspect of human movement science are constantly in-depth, help to improve the performance of athletes.

\section{Human movement science and physical recovery、 nutritional supplement}

A large number of research topics in human movement science come from the recovery of physical ability and nutrition. Physical recovery includes recovery phase and over recovery phase. Once the training class is over, it is in the recovery stage, the body begins to replenish the consumed energy, repair the damage and restore the internal environment. The length of time required for recovery depends primarily on the degree of fatigue. The deeper the degree of fatigue, the longer it takes to recover, and vice versa. In addition, systemic fatigue recovery rate is relatively slow. The main factors that influence the recovery of exercise load are as follows: (1) the longer the body tolerance phase, the deeper the fatigue, the longer the recovery time, vice versa. (2) the complement of energy after the end of practice is more timely, the more the recovery measures are in place, the faster the recovery, vice versa. After training if the arrangement has enough time to recover, in the body structure and function reconstruction, energy consumption of the sport and the reduced body function can not only recover, and will exceed the original level, this phenomenon is called "excess compensation" or "excess recovery”. General will also be due to excessive compensation caused by the improvement of the function known as the training effect. It is our training goal to produce as much training as possible. It is the most important criterion to evaluate whether the training is effective, whether the body can get enough recovery and the phenomenon of excessive compensation. The phenomenon of excessive recovery indicates the improvement of exercise capacity. At this time, if the body to fight against the same as the previous load stimulation, the body reaction will become smaller, or be able to achieve greater physical load.

Rational nutrition is an important factor in sports ability and health. Exercise can accelerate the metabolism of matter and energy, and put forward higher requirements for nutrition. There are some substances which are closely related to the exercise ability in the nutrition activities of athletes. They play an important role in regulating the function of body, accelerating the elimination of fatigue and promoting the recovery of function. These substances include phosphoric acid, creatine, sugar, vitamins, minerals and traditional Chinese medicine. The means to promote the elimination of sports fatigue mainly include finishing activities, sleep, nutrition, medical physical and psychological rehabilitation means. 


\section{Human movement science and medical supervision}

As a branch of medicine, sports medicine as an independent subject, it has a history of 54 years. With the rapid development of medical science and electronic technology, as well as the extensive development of sports, sports medicine, which is a new discipline, it has been enriched and added new content. Sports medicine is an applied science, which is a combination of medical knowledge and sports. Of course, sports medicine itself has its basic theory, such as sports anatomy, sports physiology,

Such biochemistry. However, a considerable part of these disciplines also belong to the scope of application. As for sports injuries, medical supervision, etc., are mainly in the scope of application. Medical supervision is an important part of sports medicine. It is a scientific study on the health status, exercise ability and its influencing factors. The science research mainly includes sports injury prevention and treatment of sports medicine, orthopedics, trauma, treatment and protection of athletes, wound protection, treatment and recuperation recuperation, athletes common movement disorders treatment and rehabilitation, health protection and health care and rehabilitation of injury protection technology, common sports health care knowledge and reasonable rehabilitation of athletes the nutritional content, practical and very readable. These are also the long-term concern and research content of sports science, and this is also a problem in competitive sports.

\section{Human movement science、fatigue monitoring and elimination}

Sports fatigue mainly includes three aspects: (1) the fatigue caused by special exercises. This kind of fatigue is essentially physiological fatigue. (2) the large area fatigue caused by the total training stimulation in the training course. This kind of fatigue is mainly psychological fatigue. (3) the fatigue accumulation or long-term fatigue caused by athletes during a training period. This fatigue includes both physical and psychological fatigue, including biomechanical fatigue. Coaches and athletes should pay attention to these fatigue, promote them to eliminate as soon as possible. The degree of fatigue is proportional to the time required for recovery. The deeper the fatigue is, the longer the recovery time is. However, the recovery time can not be too long, so as to avoid the movement of the effect of extinction. The training process is actually a repeated process of destruction and reconstruction of the structure and function of the body. There will be fatigue training, specific training to what time to fatigue, fatigue to what extent is determined by the training program. The ultimate goal of training is to get the training effect, but there is no functional recovery without fatigue, it will not get the training effect. After the exercise fatigue, without any means of elimination will be slowly eliminated. But the training is continuous, there is not enough time for the body to eliminate fatigue, using different means to promote the elimination of fatigue has become a research hotspot.

\section{Human movement science and doping control research}

Doping control refers to the planning, implementation and management of the work, including the detection of distribution plans, urine collection and processing, laboratory analysis, results management, hearings and complaints and other matters. The organizing committee may set up a special department to deal with doping control affairs. Doping in sports refers to the general provisions of the international sports organization of prohibited substances and disable methods. Not only the drugs that have the function of excitement, but also many other drugs. Such as diuretics, and even inhibition of solvent. It also includes a number of non drug substances, such as drugs that have never been used or other substances that are not listed on the national drug list. The 
method also includes physiological and other substances, such as blood, urine and contain banned drug ingredients of food additives, nutritional supplements, beverages, as long as these physiological substances with non normal or abnormal way through the intake of the human body, it is also a stimulant. Another example of blood transfusion with red blood cells and enhance the value of the urinary bladder input to evade detection methods are the use of stimulants. In some foods, drinks, supplements contain a certain ban than the use of chemical composition and beyond the limit, but also stimulants. The research and development of these stimulants can not be separated from the research of human movement science.

\section{Conclusion}

To enhance the physical quality of the students is the most important task for the college physical education, strengthen the use of exercise prescription is to guide students in professional sports, and should be able to according to their own development characteristics and conditions of making motion corresponding case, students can have a purpose and plan of physical exercise. The combination of health and exercise, and in the formulation of exercise prescription, rely on the human movement science, which is the most basic theory for exercise prescription. In this process, human movement science has played a role in the formulation of exercise prescription.

Human movement science on the culture in lifelong sports concept formation and advantage in sports teaching process in colleges and universities in the past, mainly on the sports action guide, because the action of complexity so students in master have great difficulty, and it is difficult to stimulate interest in learning physical education, it has not been reflected system of sports knowledge. The students in the sports scientific participation are also affected, but the application in sports human science can let students in the sports learning process, to have a better understanding of sports and human body, help students master the system of sports scientific knowledge, and form the correct sports theory. Not only can get the school sports knowledge and skills to get good practice, even after graduation can also exercise the right way to achieve the goal of lifelong physical exercise.

Physical education in colleges and universities on application of sport biomechanics in human movement science can also play a positive role in the development of sports biomechanics, some college P.E. Teachers' understanding of the subject is more detailed, its importance has also been improved. It is very important to apply the principle of sports biomechanics in the teaching of physical education for students, which can make students take the initiative to complete the creative action technology. All in all, the application of human movement science in college sports should be paid more attention, and the latest development of human movement science can be effectively combined.

\section{References}

[1] Zhang Y B. Frontier practice of functional movement training in competitive sports[J]. Shandong Sports Science \& Technology, 2012.

[2] Luo M, Tu Y. Application of Trimp in Training Monitoring of Competitive Sports[J]. Open Cybernetics \& Systemics Journal, 2015, 9(1):2463-2466.

[3] Zhang Y. Stability and Mobility in Human Movement Patterns[J]. China Sports Coaches, 2012.

[4] Yan-Ling L I. On the functions of market to the operation of Competitive Sports[J]. Journal of Educational Science of Hunan Normal University, 2002. 
[5] Tang Q, Bian B, Zhu N, et al. The Application of Training Impulse (TRIMP) in Marathon Training Load Monitoring[J]. Sports \& Science, 2013.

[6] Zhu N, Sheng L, Cao P. The Application on Trimp in Sports Monitoring[J]. Journal of Sports \& Science, 2011.8.

[7] Foster C, Florhaug J A, Franklin J, et al. A new approach to monitoring exercise training.[J]. Journal of Strength \& Conditioning Research, 2001, 15(1):109-115. 